Résumés des conférences et travaux

\title{
Épigraphie latine du monde romain
}

\section{François Bérard}

\section{(2) OpenEdition \\ Journals}

Édition électronique

URL : https://journals.openedition.org/ashp/4294

DOI : $10.4000 /$ ashp.4294

ISSN : 1969-6310

Éditeur

Publications de l'École Pratique des Hautes Études

\section{Édition imprimée}

Date de publication : 1 septembre 2021

Pagination : 180-188

ISSN : 0766-0677

Référence électronique

François Bérard, "Épigraphie latine du monde romain ". Annuaire de l'École pratique des hautes études (EPHE), Section des sciences historiques et philologiques [En ligne], 152 | 2021, mis en ligne le 14 juin 2021, consulté le 15 juin 2022. URL : http://journals.openedition.org/ashp/4294 ; DOI : https://doi.org/ 10.4000/ashp.4294 


\title{
ÉPIGRAPHIE LATINE DU MONDE ROMAIN
}

\author{
Directeur d'études : M. François BÉRARD
}

Programme de l'année 2019-2020 : I. Le dossier épigraphique de la presqu'île lyonnaise, en particulier du sanctuaire des Trois Gaules. - II. Inscriptions concernant l'administration de l'empire romain et le monde des cités.

\section{Le dossier épigraphique de la presqu'île lyonnaise, en particulier du sanctuaire des Trois Gaules}

Le sujet abordé cette année concerne à nouveau les provinces gauloises, dont la conférence avait étudié l'administration au cours des années précédentes, mais dans une perspective très différente, puisqu'il s'intéressera principalement aux inscriptions de Lyon, ou plutôt de la partie de la ville située entre le Rhône et la Saône. Il s'agira de revoir l'ensemble du matériel épigraphique, qui est relativement important, même s'il est moins riche que celui que l'on trouve sur la rive droite de la Saône et sur la colline de Fourvière, où était établie la colonie romaine. Ce travail s'inscrit dans le cadre de la préparation de deux volumes importants, le corpus épigraphique de la colonie de Lyon et l'Atlas topographique de Lugdunum, dont le deuxième tome traitera des quartiers fluviaux et de la presqu'île, le premier, paru en 2018, ayant été consacré à la colline de Fourvière. Les inscriptions les plus nombreuses et les plus intéressantes sont celles qui provenaient du sanctuaire fédéral des trois provinces de Gaule, établi sur les premières pentes de la colline de la Croix-Rousse, mais dont les pierres ont été remployées dans les églises, les caves et les ponts de la presqu'île. Cela nous amènera à nous intéresser principalement à l'organisation du sanctuaire et au rôle qu'il jouait, depuis sa fondation par Drusus en 12 av. J.-C., dans la célébration du culte impérial. Mais les questions administratives ne seront pas pour autant totalement absentes, car on semble bien avoir honoré aussi dans le sanctuaire des gouverneurs et des procurateurs, conformément au rôle politique qui était celui du concilium Galliarum. Et on s'occupera aussi des inscriptions qui ne venaient pas du sanctuaire et qui nous font connaître d'autres secteurs de la société lyonnaise, en particulier celui de l'artisanat et du commerce, dont les activités étaient surtout implantées à proximité des fleuves, dans les fameuses canabae.

On a commencé l'enquête par le quartier Saint-Vincent, qui s'étend sur la rive gauche de la Saône au pied de la colline de la Croix-Rousse. On a trouvé là au début du $\mathrm{XIX}^{\mathrm{e}}$ siècle un autel offert à Diane Auguste $(C I L, \mathrm{XIII}, 1670=\mathrm{A}$. Allmer et P. Dissard, Inscriptions antiques du musée de Lyon, II, 109), qu'on attribuait traditionnellement à un temple de Diane, et qu'il faut peut-être mettre désormais en rapport avec un monument public, doté d'une abside et d'installations thermales, dégagé alors de fouilles conduites dans le voisinage immédiat dans les années 1980. On pourrait avoir là un sanctuaire, ou un ensemble de sanctuaires, auquel il est tentant de rattacher aussi un petit autel à Maia découvert à proximité (CIL, XIII, $1748=A D$, III, 199). Mais le plus 
important est que la dédicace à Diane est offerte in honorem pagi Condatensis par le magister du pagus, nommé C. Gentius Ollilus, à un emplacement donné par un décret du pagus. Elle était donc probablement au siège de ce pagus, qui devait se trouver au bord de la Saône toute proche, comme l'indique le nom celtique de Condate, qui désigne un confluent. Il s'agit là d'une indication toponymique précieuse, l'autre attestation épigraphique du nom restant beaucoup plus incertaine (CIL, XIII, $1684=A D$, II, 130; voir infra). Si le sens du mot ne fait pas de doute, l'appartenance politique de ce pagus a suscité de nombreux débats : plutôt qu'un territoire indépendant, sur lequel aurait été établi le sanctuaire fédéral, ou qu'une partie de la cité des Ségusiaves, il vaut sans doute mieux y voir un pagus de la colonie de Lyon, pourvu, comme c'était l'usage, de ses propres institutions. Les noms celtiques, au demeurant très rares, du magister ne sont nullement incompatibles avec une citoyenneté lyonnaise, comme le montrent les noms de plusieurs décurions ou magistrats de la colonie. Quant aux rapports que le pagus de Condate pouvait avoir avec le sanctuaire voisin, c'est une question qui reste obscure, mais la découverte d'habitations laisse penser que le quartier Saint-Vincent pouvait se trouver à l'extérieur du sanctuaire.

Pour mieux comprendre le cas du pagus de Condate, on a ensuite examiné les autres pagi connus dans le voisinage et dans la province de Lyonnaise, comme ceux de Bussy-Albieux, chez les Ségusiaves, de Belley et de Briord, dans l'Ain, ou plus loin ceux des Riedones (CIL, XIII, 1646, 2544 et ILTG, 303, 3148-3152 et AE, 19691970, 405; cf. M. Tarpin, Vici et pagi dans l'Occident romain, p. 410-413). On s'est particulièrement intéressé aux autres décrets de pagi recensés en Gaule (CIL, XIII, 2608-2609, 4636; cf. 4679), ainsi qu'aux quelques exemples de magistri pagi connus (CIL, XIII, 5, 412, 604, 2507, 4316); cf. M. Dondin-Payre, dans Cités, municipes, colonies, p. 211 et 214-215.

On reconnaît en général une seconde mention du pagus de Condate dans une autre inscription lyonnaise, une grande base monumentale qui a été découverte lors de la démolition de l'église Saint-Côme, dans la presqu'île, non loin de l'église SaintPierre $(C I L$, XIII, $1684=A D$, II, 130). Cette localisation et la disposition du champ épigraphique en deux colonnes suffisent à montrer qu'il s'agit d'une base multiple honorant un prêtre des Trois Gaules en compagnie d'autres membres de sa famille, comme on en a beaucoup d'autres exemples : la seconde colonne, dont il ne reste que les premières lettres, pouvait ainsi contenir le nom de la fille du prêtre, comme l'ont proposé à juste titre Allmer et Hirschfeld, et peut-être celui d'un petit-fils, s'il faut bien lire ma[tri] à la ligne 7. Si le nom du prêtre a disparu avec le haut de l'inscription, son titre figure à la dernière ligne de la première colonne, et sous la deuxième colonne, la partie supérieure d'un T confirme que le monument, qui devait être beaucoup plus large, était dédié par les trois provinces de Gaule. Mais curieusement ce T, qui avait été parfaitement compris par le premier conservateur du musée, Artaud, n'a pas été repris dans les corpus ultérieurs de A. de Boissieu, Allmer et Hirschfeld.

La carrière municipale contenue dans la première colonne ne peut pas être, comme l'ont cru les premiers éditeurs, lyonnaise, mais doit être celle d'un magistrat d'une cité gauloise, selon toute vraisemblance, comme l'a proposé Hirschfeld au CIL, celle des Sénons, où $\mathrm{C}$. Amatius Paterninus accomplit un cursus très semblable, avec 
notamment les mêmes charges d'actor publicus et de duumuir ab aerario (CIL, XIII, 2949 = ILS, 7049; cf. M. Dondin-Payre, Cités, municipes, colonies, p. 180-181, selon laquelle l'attribution aux Sénons reste cependant hypothétique; J. Guerrier-Delclos, dans $C A G, 89,1,2002$, p. 114). Comme l'a noté Hirschfeld, un argument supplémentaire pourrait être fourni par le texte de la deuxième colonne, si du moins il faut bien restituer à la 1. 6 l'adjectif Senonius, ce qui reste incertain. La première fonction conservée en haut de la première colonne, celle de praef(ectus) coloniae, fait difficulté. S'il s'agit déjà de la cité des Sénons, il faut supposer sinon une erreur, du moins une expression approximative, à moins de lui attribuer un statut de colonie latine qui n'est pas attesté par ailleurs; mais on peut éventuellement envisager une autre colonie dont le nom figurerait plus haut, comme Hirschfeld, qui suggère celle des Vellaves. C'est immédiatement auparavant, dans la première moitié de la première ligne, qu'on restitue en général, depuis Allmer, Hirschfeld et déjà Mommsen, le titre de patr[on(o) pagi Cond]ate. Avec 10 lettres, il remplit parfaitement la lacune, mais la restitution est largement hypothétique, puisqu'il ne reste que les deux dernières lettres du nom.

Après la carrière municipale, couronnée par le flaminat du culte impérial, est mentionnée la procuratèle de la province d'Hadrumète, en Afrique proconsulaire, avec, précise le texte, un salaire de 100000 sesterces. L'anonyme est un des cinq procurateurs connus pour ce district de l'administration patrimoniale de la province d'Afrique, ordinairement appelé regio (ou diocesis) et pour lequel le mot prouincia paraît un peu inexact (voir CIL, VIII, 7039, 23068, 11174 et 23219, les deux derniers avec un rang ducénaire; Pflaum, Carrières, III, p. 1094, et M. Christol, dans CCG, 17, 2016, p. 226-228). Cet unique poste procuratorien laisse penser que la carrière est probablement ascendante, la prêtrise fédérale en constituant comme le couronnement. Mais il fournit aussi une datation au moins approximative, le diuus Aurelius Antoninus n'étant sans doute pas Marc Aurèle, comme on l'a longtemps pensé malgré l'absence du prénom Marcus, mais plutôt, ainsi que l'ont justement noté D. Fishwick (ICLW, I, p. 324-325, et III, 2, p. 67, n 32) et M. Christol, Caracalla, qui fut divinisé sous le règne d'Élagabal. Cette chronologie plus tardive s'accorde également mieux avec la mention du salaire, qui, comme l'a rappelé M. Christol, n'apparaît guère avant la fin du $\mathrm{II}^{\mathrm{e}}$ siècle et ne se développe vraiment qu'au $\mathrm{III}^{\mathrm{e}}$, et surtout avec la titulature $a d$ aram Caesaris n(ostri), dont D. Fishwick a bien montré qu'elle était caractéristique de l'époque sévérienne.

S'il avait exercé auparavant, de manière assez exceptionnelle, une procuratèle, l'anonyme sénon n'en était pas moins honoré en tant que prêtre fédéral, et sa base s'inscrit parmi la dizaine de monuments familiaux dont nous avons conservé une trace dans ce quartier des Terreaux. La plupart ont été trouvés en remploi dans les églises, les caves des maisons ou les piles des ponts, mais l'un d'entre eux a été découvert en place, en 1859, lors de la démolition des bâtiments de l'Aumône générale et de la construction de l'hôtel du Parc, près de l'angle nord-ouest de la place des Terreaux. Cette découverte exceptionnelle a été très bien documentée par le conservateur du musée de l'époque, E. Martin Daussigny, dont les données très précises ont été reprises par F. Richard, dans un article paru dans les Mélanges Robert Turcan en 1999, p. 383-394. Il s'agit de trois blocs de calcaire formant une base qui 
étaient encore sur leur lit de pose, à l'angle nord-ouest de l'emprise, sous l'actuelle rue Sainte-Catherine, et à proximité desquels ont été découverts huit autres blocs, dont trois, portant une inscription, appartenaient à la partie centrale du monument, et cinq, moulurés, à la corniche (CIL, XIII, $1692=A D$, II, 133). La principale caractéristique de ces blocs est leur forme concave, qui permet de reconstituer un monument en forme d'hémicycle, qui avait jadis été remonté dans la grande réserve épigraphique du musée de Fourvière; les dimensions sont considérables, puisqu'on atteint 2,55 m de hauteur, au-dessus desquels il faut placer les statues, pour une largeur que le rayon de courbure permet d'estimer à environ $8 \mathrm{~m}$. Une première inscription, qui figure sur les deux premiers blocs, mais commençait sur un bloc voisin qui n'a pas été conservé, est une dédicace faite par les trois provinces de Gaule à [Iu]lia Salica, épouse de [Ti. E]ppius Bellicus. Le troisième bloc conserve le début d'une nouvelle colonne, qui devait se poursuivre sur les blocs suivants et où on reconnaît à la deuxième ligne le prénom Ti(berius) et à la troisième le début du mot $s a[c e r d o s]$, ce qui laisse penser qu'il pouvait s'agir d'un autre parent du même prêtre fédéral. L'hémicycle a donc très vite été identifié comme le monument familial d'un prêtre sortant de charge, d'autant que le nom de Ti. Eppius Bellicus se retrouve dans une dédicace aux numina Augustorum aujourd'hui perdue, mais qui a été copiée dans l'église Saint-Pierre par les principaux antiquaires du $\mathrm{XVI}^{\mathrm{e}}$ siècle (CIL, XIII, 1678). À l'est de l'hémicycle ont été dégagées trois autres bases monumentales, mais rectilignes et dépourvues d'élévations. Elles sont donc également dépourvues d'inscriptions, mais on a trouvé devant la deuxième et là aussi en place un autel offert aux numina Augustorum par un nommé Ti. Claudius Genialis (CIL, XIII, $1677=A D$, II, 137). La découverte a permis de comprendre que ces bases familiales étaient accompagnées d'un autel offert par le prêtre sortant de charge, comme l'avaient très bien vu Allmer et déjà en partie Martin Daussigny. Un autre intérêt de ce très beau dossier est de montrer que le sanctuaire fédéral s'étendait au moins jusqu'aux abords de la place des Terreaux, au pied de la colline de la Croix-Rousse.

Un autre monument familial est celui auquel appartenait la base du jeune décemvir stlitibus iudicandis C. Iulius Taurus, découverte en 1849 dans une cave rue Paul Chenavard, en face de la rue Saint-Côme, c'est-à-dire tout près de Saint-Pierre (CIL, XIII, $1682=A D, \mathrm{I}, 19)$. Comme l'a bien vu Allmer, cette base devait figurer dans le monument offert par les Trois Gaules à un parent, sans doute un père ou un oncle, qui était prêtre fédéral. Celui-ci devait figurer dans la partie gauche du monument, avec peut-être aussi son épouse; mais il est impossible de savoir si le monument comptait d'autres membres de la famille et d'estimer en conséquence sa taille. Le rang sénatorial est exceptionnel dans le milieu des prêtres des Trois Gaules, mais il y a cependant un parallèle, là aussi à la seconde génération, dans la base de M. Marius Florentinus, qui était le frère d'un prêtre fédéral, mais se définit aussi comme pater senatoris (CIL, XIII, $1683=A D$, II, 136; cf. F. Bérard, dans Hommage à Jean-Louis Ferrary, Paris, 2019, p. 458-460). On ne sait si le jeune homme avait aussi une statue, comme C. Iulius Taurus, dans le monument de son oncle, mais l'honneur rejaillissait de toutes façons sur l'ensemble de la famille. Le parallèle est d'autant plus justifié que la pierre a été découverte dans la démolition du Pont du Change, en 1846, et devait donc provenir elle aussi du sanctuaire, et peut-être même du quartier voisin de Saint-Pierre et de Saint-Côme. 
La vieille église Saint-Pierre, conservée dans le superbe monastère reconstruit au $\mathrm{XVII}^{\mathrm{e}}$ siècle par les Bénédictines et devenu ensuite musée des Beaux-Arts, était un

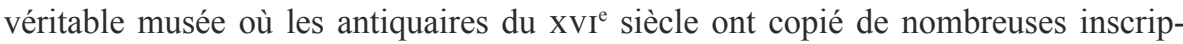
tions, dont certaines ont aujourd'hui disparu. Outre celles qui étaient à l'intérieur de l'édifice, comme la dédicace de Ti. Eppius Bellicus aux numina Augustorum (voir supra), d'autres se trouvaient à l'extérieur et l'une servait de base à la croix du cimetière qui se trouvait entre Saint-Pierre et l'église voisine de Saint-Saturnin (CIL, XIII, $1691=A D$, II, 116). Il s'agit de l'inscription de C. Catullius Deciminus, un Tricasse qui avait été sacerdos ad templum Romae et Auggg(ustorum), à l'époque sévérienne, comme le montre la mention de trois Augustes. Si cette pierre, haute de 1,66 m et aujourd'hui conservée au musée, figure dans à peu près tous les recueils épigraphiques lyonnais jusqu'au XVIII ${ }^{e}$ siècle, Bellièvre est un des seuls à signaler que le texte se poursuivait sur un deuxième bloc remployé dans le bas du clocher de Saint-Pierre, sur lequel figurent les deux ou trois dernières lettres de chaque ligne, que les copies ultérieures indiquent simplement à la suite des précédentes, et il faut consulter les recensions de Giocondo et du Florentin Symeoni ou le corpus de Gruter pour savoir que le même bloc portait ensuite une deuxième colonne où se lisait le nom de Iunia / Domitiola, / uxor / Catulli / Decimini, qui avait échappé à Bellièvre. Ces sources italiennes étant beaucoup moins accessibles, on s'explique que la base de Deciminus n'ait pas été identifiée comme monument familial avant, là encore, le corpus d'Allmer. La conclusion aurait cependant pu être tirée des deux grandes lettres TR de la dernière ligne, qui, étant hautes de $32 \mathrm{~cm}$, montrent que la dédicace $\operatorname{tr}$ [es prouinciae Galliae] devait s'étendre sous plusieurs autres blocs. Mais cette lecture n'était en général pas retenue, soit parce qu'on préférait des abréviations du type t(itulum) r(estituerunt), soit simplement parce que les trois provinces étaient déjà mentionnées à la ligne précédente. Peut-être par analogie avec le monument d'Eppius Bellicus, on restitue en effet en général à la ligne 8 le nom des (tres) pr[ouinc(iae) Galliae] au nominatif, à la suite notamment de L. Renier ou d'Allmer, qui supposait l'existence de deux dédicaces différentes, une pour la statue de Deciminus à la ligne 8 et une autre pour l'ensemble du monument à la ligne 9. Mais il paraît plus simple d'y voir, comme D. Fishwick (ICLW, III, 1, p. 200 et III, 2, p. 66, n 31), un génitif et de comprendre donc sacerd(oti) ... (trium) pr[ouinc(iarum) Galliarum], la dédicace n'occupant alors logiquement que la dernière ligne. D'autre part, même si à partir du XIX ${ }^{\mathrm{e}}$ siècle certains savants comme Artaud ou de Boissieu avaient reconnu que cette dernière ligne monumentale portait bien la dédicace des Trois Gaules, ce n'est pas avant Allmer que la structure de ces monuments familiaux comportant plusieurs statues a vraiment été comprise. Outre les monuments de Ti. Eppius Bellicus et de C. Iulius Taurus, un autre parallèle éclairant a été la grande base du Ségusiave C. Ulatt[ius ---], découverte en 1867 rue Lanterne et dont la dernière ligne porte, comme celle de C. Catullius Deciminus, les deux seules lettres TR, en caractères de $29 \mathrm{~cm}(C I L$, XIII, $1712=A D$, II, 111).

Mais la démolition du Pont du Change, en 1846, avait déjà apporté un autre élément de monument familial, avec la grande base du Ségusiave P. Maglius Priscian(us) $(C I L, \mathrm{XIII}, 1701=A D$, II, 112). La dernière ligne donne cette fois la fin de la dédicace avec les lettres [Gal]liae gravées en caractères de $27 \mathrm{~cm}$. Mais il faut 
évidemment restituer avant les mots [III prouinciae] et donc supposer l'existence de plusieurs autres inscriptions. L'une était certainement celle de Pama Priscian(?), dont P. Maglius Priscian(us) se déclare le père. Allmer envisage qu'il puisse s'agir du prêtre lui-même, qui porterait un nom masculin en $-a$, mais un nom féminin paraît beaucoup plus probable, d'autant qu'il est déjà attesté pour la sœur d'un cavalier biturige (CIL, III, 2065 ; cf. OPEL, III, p. 122) : Pama Priscian(a) pourrait être alors l'épouse du prêtre fédéral et $\mathrm{P}$. Maglius Priscian(us) son beau-père. Le nom est de toutes façons celtique, comme le montre aussi le gentilice dérivé Pamius, qui est attesté à Lyon (CIL, XIII, 11178). Il est possible que ce gendre inconnu ait été lui-même ségusiave, comme son beau-père et comme plusieurs prêtres fédéraux qui appartenaient à la famille des Ulatii (CIL, XII, 1851 et XIII, 1712), mais ce ne peut évidemment être une certitude. Le monument comportait donc au moins trois statues, celle du prêtre, celle de son épouse et celle de son beau-père, mais peut-être y en avait-il quelques-unes de plus. La place du beau-père à l'extrémité droite confirme que dans ce type de monument collectif, la statue du prêtre sortant de charge devait être à gauche, suivie de celle de son épouse, puis de ses parents proches, les parents plus éloignés se trouvant du côté droit. Mais le nombre de statues reste difficile à estimer et pouvait du reste varier d'une famille à une autre.

\section{Inscriptions concernant l'administration de l'empire romain et le monde des cités}

La deuxième heure de la conférence a d'abord été consacrée à l'étude de cursus sénatoriaux anciens, augustéens ou tibériens. Nous avons commencé par celui de Q. Varius Geminus, le premier sénateur pélignien, comme le dit fièrement l'inscription qui lui a été élevée dans sa patrie de Superaequum (CIL, IX, 3306). Un des traits caractéristiques de ces cursus anciens est le fait qu'on se contente d'indiquer le titre du dédicataire, sans préciser les provinces ou les unités militaires dans lesquelles il a exercé la fonction. On a remarqué aussi la charge de curator aedium sacrarum monumentorumque publicorum tuendorum, qui annonce celle des futurs curatores aedium sacrarum, operum locorumque publicorum, mais avec un titre et un niveau hiérarchique différents, puisque le Pélignien était encore prétorien quand il l'a exercée. On trouve une sobriété encore plus marquée dans la dédicace offerte à l'époque augustéenne par la cité de Canusium à un des ses patrons, Q. Articuleius Regulus, simplement défini comme $\operatorname{pr}($ aetor), sans cursus détaillé, et proco(n)s(ul), leg(atus) Imp(eratoris) Caes(aris) Aug(usti), sans indication des provinces (CIL, IX, $331=$ Epigrafi romane di Canosa, I, 22).

Parmi ces exemples de cursus sobres, sans indication des provinces et des légions, nous avons plus particulièrement étudié ceux de $\mathrm{T}$. Trebellenus Rufus à Concordia, leg(atus) Caesaris Augusti auquel la plèbe érigea une statue équestre (CIL, V, 1878; cf. $P I R^{2}$, T 308), à Padoue de Sex. Papinius Allenius, qui avait été deux fois leg(atus) Ti(berii) Caesaris, sans doute d'une légion, puis d'une province qui ne sont pas précisées (CIL, V, 2823; cf. $P I R^{2}, \mathrm{P} 102$ ), à Assise de Post(umus) Mimisius Sardus, leg(atus) Ti(berii) Caesaris Aug(usti), puis proconsul d'une province inconnue (CIL, XI, 3598; cf. PIR ${ }^{2}, \mathrm{M}$ 592), et enfin à Brescia de C. Pontius Paelignus, qui avait été 
legatus pro pr(aetore) iterum, ex s(enatus) c(onsulto) et ex auctoritate Ti. Caesaris, précision bizarre qui pourrait renvoyer à une situation particulière dans une province sénatoriale où il aurait été légat du proconsul (CIL, V, $4348=I I t, \mathrm{X}, 5,138$; cf. PIR ${ }^{2}$, P 813; W. Eck, dans ZPE, 106, 1995, p. 249-251). L'autre originalité de la carrière de C. Pontius Paelignus est la fonction de curator locorum publicorum, qu'il a exercée à deux reprises entre la questure et l'édilité.

Cette commission de cinq membres, dont un président de rang consulaire, qui avait pour charge de défendre la propriété publique contre les abus des particuliers, est caractéristique du début du règne de Tibère, pour lequel 4 ou 5 collèges différents sont connus (cf. A. Daguet-Gagey, "Auguste et la cura operum publicorum », dans H.-G. Pflaum, un historien du XXe siècle, p. 441-443) et semble disparaître ensuite, peut-être intégrée dans la cura aedium sacrarum, operum locorumque publicorum. Le nom de C. Pontius Paelignus apparaît sur quatre cippes, deux de collèges présidés par T. Quinctius Crispinus Valerianus, entre 19 et 23 apr. J.-C. (CIL, VI, 1266 et sans doute 40883 ; cf. G. Alföldy, CIL, VI, 8, 2, p. 4597) et deux placés sous l'autorité de L. Nonius Asprenas, dans lesquels il figure en dernière position (CIL, VI, 31754 et 37037, vers 24 apr. J.-C.). Par comparaison, on a aussi examiné trois autres collèges présidés par L. Nonius Asprenas (CIL, VI, 1267 a-b et 31753), dans lesquels ne figure pas C. Pontius Paelignus, mais où l'on trouve en revanche P. Viriasius Naso, un proconsul de Crète célèbre pour avoir fait une dédicace au numen et à la providence de Tibère après la mort de Séjan (CIL, III, 12036 = I Cret, IV, 272; cf. PIR², V 700).

Après ces cursus augustéens et tibériens, qui concernaient presque tous des sénateurs originaires d'Italie centrale, nous avons examiné quelques carrières de sénateurs provinciaux, légèrement plus tardives, puisqu'elles datent du règne de Claude. La première est celle de [T.] Domitius Decidius, connu par une inscription de Rome (CIL, VI, 1403), mais que sa tribu Voltinia et sa qualité probable de beau-père d'Agricola, dont l'épouse s'appelait Domitia Decidiana, conduisent à considérer comme originaire de la province de Narbonnaise, peut-être de la cité de Vienne (cf. M. Corbier, Aerarium, no 20; Y. Burnand, Primores Galliarum, p. 86-87). L'inscription romaine nous apprend qu'après le modeste triumvirat capitalis, qui invite à voir en lui un nouveau sénateur, il fut un des premiers questeurs nommés pour trois ans à la tête de l'aerarium de Saturne : illustration parfaite d'un excursus célèbre de Tacite (Ann., XIII, 29) qui nous apprend que Claude avait rendu à des questeurs et donc au sénat cette administration qu'Auguste avait confiée à des préfets nommés par lui qui seront ensuite rétablis par Néron. En ce qui concerne son origine, nous avons ensuite rappelé les deux inscriptions du chevalier et duumvir viennois T. Pompeius Albinus, qui après une milice avait été, avec le titre de subprocurator, adjoint d'un procurateur de la province de Lusitanie nommé T. Decidius Domitianus, dont les rapports avec le beau-père d'Agricola restent imprécis (CIL, XII, $2327=I L N$, Vienne, 515 et $A E$, 1935, 5, à Mérida; cf. S. Demougin, Prosopographie, n 562, et Y. Burnand, Primores Galliarum, II, p. 209-211).

Nous avons par chance conservé le cursus d'un autre de ces éphémères quaestores aerarii, nommé L. Coiedius Candidus (CIL, XI, 6163; cf. M. Corbier, Aerarium, $\mathrm{n}^{\mathrm{o}} 21$, et S. Antolini, dans Suppl It, 18, p. 339-341). La chance est aussi que cette dédicace offerte par la cité ombrienne de Suasa est pourvue d'une sorte d'éloge final très 
circonstancié qui précise que le dédicataire fut nommé quaestor aerarii l'année où il était questeur de l'empereur et indiquait auparavant qu'il avait été décoré par Claude de trois couronnes et d'une haste. On rapporte en général ces décorations au tribunat militaire dans la VIII ${ }^{\mathrm{e}}$ Augusta et au soulèvement du légat de Dalmatie Scribonianus pendant lequel cette unité serait, comme les deux légions de la province, restée fidèle à Claude. Mais on peut préférer, comme A. R. Birley (Government of Britain, p. 214) et déjà Dessau, la campagne britannique de Claude, à laquelle Candidus a pu participer comme questeur de l'empereur. Un argument supplémentaire en ce sens est le montant des décorations, qui, avec trois couronnes, est en principe plutôt celui des sénateurs de rang prétorien, les tribuns laticlaves n'en ayant que deux et les officiers équestres en général une seule. Comme le tribunat de Candidus, effectué avant le vigintivirat, était probablement angusticlave (cf. en ce sens Devijver, PME, C 218), il faut rapporter les décorations à un stade ultérieur de la carrière, et donc au poste de questeur de l'empereur, ce qui ne saurait surprendre quand on connaît la générosité avec laquelle Claude décora alors son entourage; un tribunat laticlave, comme l'envisage V. Maxfield (Military Decorations, p. 152), est une solution beaucoup moins satisfaisante, parce que la VIII ${ }^{\mathrm{e}}$ Augusta ne semble pas avoir participé à l'expédition de Bretagne et surtout en raison de sa place avant le vigintivirat. Une telle révision a aussi une conséquence chronologique : comme l'éloge précise que Coiedius Candidus a été nommé questeur de l'aerarium l'année où il était questeur de l'empereur, sa direction de l'aerarium doit être placée dès 44 apr. J.-C. et non vers 50, comme le suggérait M. Corbier, et il doit alors l'avoir exercée en même temps que T. Domitius Decidius.

L'inscription de Suasa mentionne ensuite une dernière charge, celle de curator tab(ularum?) p(ublicarum?), c'est-à-dire de directeur des archives publiques, probablement distincte de la questure et postérieure à celle-ci, même si elle a bénéficié de l'expérience acquise dans cette magistrature. Le poste est caractéristique d'une époque, celle des Julio-claudiens, où les archives avaient été retirées aux préteurs ou aux questeurs de l'aerarium et confiées à des curateurs spéciaux, qui formaient un collège de trois membres, dont on connaît une attestation épigraphique pour l'année 46 apr. J.-C. (CIL, VI, $916=31201)$. Outre L. Coiedius Candidus, on possède un second exemple individuel dans le cursus de C. Vmmidius Durmius Quadratus, qui a exercé la fonction après avoir été praetor aerarii sous le règne de Tibère $(C I L, \mathrm{X}$, 5182; cf. M. Corbier, Aerarium, $\mathrm{n}^{\circ} 10$ ) : on y retrouve la même succession logique de la magistrature à la curatèle, même si c'est cette fois au niveau prétorien. L'épigraphie conserve ainsi la trace des réformes successives qui se sont succédé entre Auguste et Néron, confirmant globalement la synthèse qu'en a proposée Tacite (Ann., XIII, 28-29), même s'il subsiste quelques incertitudes dans le détail. Mais, comme l'a bien noté l'historien, ces changements prennent fin sous le règne de Néron, qui a sans doute attribué la gestion des archives financières aux préfets de l'aerarium au moment où ils furent définitivement recréés.

On a poursuivi en examinant la très belle carrière qui a conduit ensuite $\mathrm{C}$. Vmmidius Durmius Quadratus jusqu'au gouvernement de la Syrie et à la guerre contre les Parthes, où il se trouva en rivalité avec Corbulon, en s'appuyant sur le complexe cursus de Casinum, qui est de type compartimenté (CIL, X, 5182), mais aussi sur 
la nouvelle inscription découverte à Apamée $(A E, 2000,1496$; cf. J.-C. Balty, dans CRAI, 2000, p. 460-466, et $P I R^{2}, \mathrm{~V}$ 903). Ce fut l'occasion de rappeler la célèbre figure de sa fille, Vmmidia Quadratilla, qui a offert un amphithéâtre à ses compatriotes de Casinum (CIL, X, 5183) et dont le petit-fils était un des protégés de Plinele-Jeune (VII, 24). On a terminé en étudiant la carrière de ce dernier, consul en 118 et gouverneur de Mésie inférieure entre 119 et 121 (IScM, II, 48; cf. PIR², V 907).

Enfin, à propos d'un prêtre anonyme sénon qui l'avait exercée sans doute avant d'arriver à la prêtrise fédérale (voir supra, CIL, XIII, 1684), on s'est intéressé à la procuratèle de la région d'Hadrumète, qui était une des trois circonscriptions patrimoniales de l'Afrique proconsulaire. Le dossier, qui a été réexaminé dans un article récent de M. Christol (CCG, 17, 2016, p. 226-228), compte cinq titulaires connus : outre le Sénon, CIL, VIII, 7039, 23068, 11174 et 23219; cf. Pflaum, Carrières, III, p. 1094. Deux d'entre eux le sont par des inscriptions de Segermes, qui ont été reprises dans le récent catalogue de L. L. Sebai ( $\mathrm{n}^{\text {os }} 16$ et 18$)$. Le premier, un anonyme qui avait obtenu une quatrième milice, avait exercé auparavant deux petites procuratèles et était donc probablement encore centenaire, comme le prêtre sénon. Les inscriptions du second, C. Postumius Saturninus Flauianus, précisent en revanche qu'il avait été promu sur place du rang centenaire à celui de ducénaire, sans doute pas avant le milieu du III $^{\mathrm{e}}$ siècle (CIL, VIII, 11174-11175), et on peut envisager une date analogue pour le second ducénaire, $[\mathrm{P}]$ omponius L[...]murianus, qui avait été auparavant avocat du fisc et a commentariis des préfets du prétoire (CIL, VIII, 23219). Dans toutes ces inscriptions, la circonscription d'Hadrumète est régulièrement appelée regio ou diocesis, confirmant que l'emploi de prouincia dans l'inscription lyonnaise est sans doute un peu approximatif. 